\title{
Relación entre los Valores Bioquímicos Séricos Fetal y Maternal en Alpacas
}

\author{
Relationship between Foetal and Maternal Serum Biochemistry in Alpacas \\ José Rodríguez G. ${ }^{1}$, Manuel Barrios-Arpi ${ }^{2,5}$, Bernardo Lopez-Torres ${ }^{1}$, \\ Alejandro Rodríguez G. ${ }^{3}$, Luis Revuelta Rueda ${ }^{4}$
}

\section{Resumen}

El objetivo del estudio fue relacionar los valores bioquímicos séricos fetales y maternales durante los tres tercios de gestación de la alpaca (Vicugna pacos). Se utilizaron 24 alpacas gestantes y sus respectivos fetos para determinar los niveles de glucosa $(\mathrm{G})$, triglicéridos $(\mathrm{T})$, colesterol $(\mathrm{C})$, proteína total $(\mathrm{PT})$, albúmina $(\mathrm{A})$, calcio $(\mathrm{Ca})$, bilirrubina total (BT), bilirrubina directa (BD) y fosfatasa alcalina (FA). El número de animales para el primer, segundo y tercer tercio de la gestación fue de 6,7 y 11, respectivamente. Las correlaciones materno-fetal fueron de G: 0.81, C: 0.70, T: 0.85, PT: 0.38, A: 0.38, FA: 0.29, Ca: 0.01 , BT: 0.15 y BD: -0.05 . Los valores bioquímicos séricos siempre fueron significativamente diferentes (a partir de $\mathrm{p}<0.05$ ) en el último tercio de gestación, tanto en fetos como en madres. Se concluye que los parámetros bioquímicos se incrementaron hacia el término de la gestación y la mayor parte de los perfiles séricos guardan una relación positiva entre las madres y sus fetos.

Palabras clave: bioquímica sérica, relación materno-fetal, alpaca

\section{AbSTRACT}

The aim this study was to relate the fetal and maternal serum biochemistry levels in the three thirds of the gestation period of the alpaca (Vicugna pacos). In total, 24 pregnant alpaca and their fetus were sampled to analyze levels of glucose $(G)$, triglycerides $(T)$,

${ }^{1}$ Estación Experimental del Centro de Investigación IVITA - El Mantaro, Huancayo, Perú

${ }^{2}$ Laboratorio de Patología Clínica y Biología Molecular, Facultad de Medicina Veterinaria Universidad Nacional Mayor de San Marcos, Lima, Perú

${ }^{3}$ SENASA, Región Junín, Perú

${ }^{4}$ Departamento de Fisiología Animal, Universidad Complutense de Madrid, España

${ }^{5}$ E-mail: manuelvet26@gmail.com

Recibido: 13 de enero de 2016

Aceptado para publicación: 22 de abril de 2016 
cholesterol (C), total protein (PT), albumin (A), calcium (Ca), total bilirubin (BT), direct bilirubin (BD), and alkaline phosphatase (FA). The number of animals in the first, second and third stage of gestation were 6,7 and 11 respectively. The maternal-fetal correlations were G: 0.81, C: 0.70, T: 0.85, PT: 0.38,A: 0.38,FA: 0.29, Ca: 0.01, BT: 0.15 and BD: -0.05 . The serum biochemistry levels were significantly different (from $\mathrm{p}<0.05$ ) mainly in the third gestation period. It is concluded that all the biochemical parameters increased during gestation and the relationship between maternal-fetal serum biochemistry was positive.

Key words: serum biochemistry, maternal-fetal realationship, alpaca

\section{INTRODUCCIÓN}

La gestación y la lactación son etapas fisiológicas que modifican el metabolismo de los animales (Krajnicakova et al., 2003; Iriadam, 2007). Durante la preñez, las alpacas (Vicugna pacos) deben tener un estado de salud óptimo para el logro de una cría viable y capaz de regular adecuadamente su metabolismo energético (Rodríguez et al., 2012). La identificación de cambios en el metabolismo delosanimal esdurantesu vidareproductiva podríallevar aidentificar y predecir al gunos desórdenes metabólicos. Los perfiles metabólicos han sido usados para predecir problemas metabólicos pre y posparto, para el diagnóstico de enfermedades metabólicas y paralaeval uación deestado nutricional del animal. Por ejemplo, la glucosa (Huaynates et al., 2016) en la alpaca es la principal fuente energética fetal, así como ocurre en el feto ovino, y sus requerimientos incrementan a medida que transcurre la gestación, debido al crecimiento del feto (Fýrat y Özpinar, 2002).

Conforme avanza la preñez, se notan las diferencias en las concentraciones séricas de glucosa, colesterol y proteína total debido a los requerimientos nutricionales de las madres (Brozostowski et al., 1995; El-Sherif y Assad, 2001; Nazifi et al., 2002). Otros parámetros bioquímicos que incluyen triglicéridos, ácidos grasos y urea son importantes indicadores de la actividad metabólica en diversos periodos reproductivos en los animales (Karapehlivan et al., 2007).
Durante la preñez, diversos tejidos maternales están involucrados en el suministro energético para el feto. Así mismo, otros elementos podrían alterar este suministro energético como la edad de la madre, la nutrición maternal, el crecimiento fetal y el medio ambiente (Swanson et al., 2004; Yokus et al., 2006).

No se conocen los valores bioquímicos sanguíneos de alpacas durante la etapa de gestación ni como estos valores influyen en el feto. Es por ello que se implementó el presente estudio para determinar los niveles séricos maternales y fetales de glucosa, triglicéridos, colesterol, proteína total, albúmina, calcio, bilirrubina total, bilirrubina directa y fosfatasa alcalina, así como su correlación entre los tres tercios de la gestación de la alpaca.

\section{Materiales y Métodos}

\section{Animales y Muestras}

Se trabajó con 24 alpacas gestantes [en el caso de la glucosa se utilizaron los datos de 18 animales del estudio de Huaynates et al. (2016)], clínicamente sanas de 3-5 años de edad, y sus respectivos fetos, que fueron colectados en el matadero municipal Ninacaca de la Región Pasco, Perú.

Los animales fueron agrupados según el tercio de gestación: 6 fetos (F1) y 6 madres (M1) en el primer tercio, 7 fetos (F2) y 
Cuadro 1. Correlación (r) entre valores bioquímicos séricos maternales y fetales de alpacas

\begin{tabular}{lc}
\hline Parámetros & Correlación \\
\hline Glucosa & $0.81^{* * *}$ \\
Colesterol & $0.70^{* * *}$ \\
Triglicéridos & $0.85^{* * *}$ \\
Proteína total & $0.38^{*}$ \\
Albúmina & $0.38^{*}$ \\
Fosfatasa alcalina & $0.29^{*}$ \\
Calcio & 0.01 \\
Bilirrubina total & 0.15 \\
Bilirrubina directa & -0.05 \\
\hline$* * *$ & \\
positiva alta; ${ }^{*}$ positiva baja &
\end{tabular}

7 madres (M2) en el segundo tercio, y 11 fetos (F3) y 11 madres (M3) en el tercer tercio de gestación. Para esto, los 345 días aproximados de la gestación de la alpaca fueron divididos entre tres y la edad fetal fue calculada según el diámetro biparietal fetal, según la fórmula propuesta por Gazitua et al. (2001).

Las muestras de sangre fetal y materna se obtuvieron por punción intracardiaca y de la vena yugular, respectivamente. El periodo de ayuno fue de aproximadamente 12 horas previas al sacrificio. La sangre materna se obtuvo antes y después del sacrificio a fin de comparar posibles alteraciones post mortem. La sangre fue colectada en tubos de ensayo sin anticoagulante y centrifugada a $3000 \mathrm{~g}$ por $30 \mathrm{~min}$ en una centrífuga automática (GEMMY, Taiwán). El tiempo desde la colección de sangre hasta la centrifugación fue de 2-5 minutos, pues se realizó en el mismo centro de beneficio. El suero resultante se almacenó a $-196{ }^{\circ} \mathrm{C}$ en tanque de nitrógeno líquido (GEMMY, Taiwán), para su posterior traslado a la Facultad de Medicina Veterinaria, Universidad Nacional Mayor de
San Marcos, Lima, donde se realizó el análisis bioquímico sanguíneo.

\section{Análisis Bioquímico Sanguíneo}

Mediante análisis bioquímico del suero sanguíneo se determinaron los niveles de glucosa $(\mathrm{G}, \mathrm{mg} / \mathrm{dl})$, triglicéridos $(\mathrm{T}, \mathrm{mg} / \mathrm{dl})$, colesterol $(\mathrm{C}, \mathrm{mg} / \mathrm{dl})$, proteína total $(\mathrm{PT}, \mathrm{g} /$ dl), albúmina (A, g/dl), calcio ( $\mathrm{Ca}, \mathrm{mg} / \mathrm{dl})$, bilirrubina total (BT, $\mathrm{mg} / \mathrm{dl}$ ), bilirrubina directa $(\mathrm{BD}, \mathrm{mg} / \mathrm{dl})$ y fosfatasa alcalina $(\mathrm{FA}, \mathrm{U} /$ I). Se utilizaron kits comerciales (FAR Diagnostics, Italia) y se siguieron las especificaciones del fabricante. La lectura se hizo en un analizador bioquímico semiautomático (Sinowa, China).

\section{Análisis de Datos}

Los niveles de G, C, T, PT, A, Ca, BT, BD y FA se presentan como promedios y desviaciones estándares (Prom $\pm \mathrm{DE}$ ). Los datos fueron analizados mediante un análisis de varianza y la determinación de diferencias entre promedios de los tres grupos (tercios) de gestación por la prueba post-hoc de Duncan con un nivel de significación del 99.9\%. Asimismo, se determinó la relación entre los valores de G, C, T, PT, A, Ca, BT, BD y FA de las madres y los fetos mediante el coeficiente de correlación de Pearson.

\section{Resultados}

No se observaron cambios en los valores séricos maternales en las muestras tomadas antes y después del sacrificio. La mayoría de las correlaciones (r) entre los valores bioquímicos séricos maternal y fetal presentaron correlaciones positivas altas $(\mathrm{G}$, C y T) o bajas (PT, A y FA), con excepción de los valores de $\mathrm{Ca}, \mathrm{BT}, \mathrm{BD}$ donde las correlaciones fueron $\mathrm{rH} \approx 0$ (Cuadro 1).

Los valores de la bioquímica sanguínea fetal (F1, F2 y F3) y maternal (M1, M2 y M3) se presentan en el Cuadro 2. 
Cuadro 2. Niveles séricos de glucosa ${ }^{1}$, triglicéridos, colesterol, proteína total, albúmina, calcio, bilirrubina total, bilirrubina total y directa, y fosfatasa alcalina fetal (F1, F2 y F3) y maternal (M1, M2 y M3) en los tres tercios de gestación en alpacas

\begin{tabular}{|c|c|c|c|c|c|c|}
\hline & $\mathrm{F} 1$ & $\mathrm{~F} 2$ & F3 & M1 & M2 & M3 \\
\hline $\begin{array}{l}\text { Glucosa } \\
(\mathrm{mg} / \mathrm{dl})\end{array}$ & $208 \pm 3.5^{\mathrm{a}}$ & $229 \pm 14.6^{\mathrm{a}}$ & $266 \pm 16.2^{\mathrm{b}}$ & $126.5 \pm 2.2^{\mathrm{c}}$ & $126.5 \pm 9.5^{\mathrm{cd}}$ & $163.2 \pm 12.3^{\mathrm{d}}$ \\
\hline $\begin{array}{l}\text { Triglicéridos } \\
(\mathrm{mg} / \mathrm{dl})\end{array}$ & $41 \pm 0.4$ & $46 \pm 3.1^{\mathrm{a}}$ & $69 \pm 8.7^{b}$ & $110 \pm 5.0^{\mathrm{b}}$ & $137 \pm 16.69^{\mathrm{c}}$ & $213 \pm 16.7^{\mathrm{c}}$ \\
\hline $\begin{array}{l}\text { Colesterol } \\
(\mathrm{mg} / \mathrm{dl})\end{array}$ & $33 \pm 0.7^{\mathrm{a}}$ & $47 \pm 7.8^{\mathrm{a}}$ & $77 \pm 11.0^{\mathrm{b}}$ & $46 \pm 1.9^{\mathrm{ac}}$ & $113 \pm 7.2^{\mathrm{bd}}$ & $119 \pm 26.0^{\mathrm{d}}$ \\
\hline $\begin{array}{l}\text { Proteína total } \\
(\mathrm{g} / \mathrm{dl})\end{array}$ & $4.20 \pm 0.02^{\mathrm{a}}$ & $4.65 \pm 0.18^{\mathrm{a}}$ & $5.01 \pm 0.69^{\mathrm{ab}}$ & $6.42 \pm 0.18^{\mathrm{bc}}$ & $6.44 \pm 0.11^{\mathrm{c}}$ & $6.51 \pm 0.92^{\mathrm{c}}$ \\
\hline $\begin{array}{l}\text { Albúmina } \\
(\mathrm{g} / \mathrm{dl})\end{array}$ & $2.67 \pm 0.02^{\mathrm{a}}$ & $3.71 \pm 0.06^{\mathrm{ab}}$ & $4.20 \pm 0.55^{\mathrm{b}}$ & $4.47 \pm 0.07^{b}$ & $4.59 \pm 0.50^{\mathrm{b}}$ & $4.67 \pm 0.76^{\mathrm{b}}$ \\
\hline Calcio (mg/dl) & $10.4 \pm 0.2^{\mathrm{a}}$ & $11.5 \pm 0.6^{\mathrm{ab}}$ & $13.4 \pm 0.9^{\mathrm{b}}$ & $11.0 \pm 0.5^{\mathrm{a}}$ & $11.0 \pm 0.6^{\mathrm{ab}}$ & $14.0 \pm 0.5^{\mathrm{b}}$ \\
\hline $\begin{array}{l}\text { Bilirrubina } \\
\text { total (mg/dl) }\end{array}$ & $0.11 \pm 0.01$ & $0.11 \pm 0.02$ & $0.11 \pm 0.01$ & $14 \pm 0.04$ & $0.15 \pm 0.04$ & $0.16 \pm 0.11$ \\
\hline $\begin{array}{l}\text { Bilirrubina } \\
\text { directa } \\
(\mathrm{mg} / \mathrm{dl})\end{array}$ & $0.03 \pm 0.03^{\mathrm{ab}}$ & $0.03 \pm 0.01^{\mathrm{a}}$ & $0.03 \pm 0.02^{\mathrm{a}}$ & $0.04 \pm 0.03^{\mathrm{ab}}$ & $0.05 \pm 0.03^{\mathrm{ab}}$ & $0.07 \pm 0.03^{\mathrm{b}}$ \\
\hline $\begin{array}{l}\text { Fosfatasa } \\
\text { alcalina (U/I) }\end{array}$ & $114 \pm 4^{\mathrm{a}}$ & $115 \pm 30^{\mathrm{ab}}$ & $115 \pm 15^{\mathrm{a}}$ & $157 \pm 36^{\mathrm{ab}}$ & $161 \pm 21^{\mathrm{ab}}$ & $166 \pm 19^{b}$ \\
\hline
\end{tabular}

\section{Discusión}

El transporte de moléculas desde la circulación maternal a la fetal ha sido ampliamente estudiado en mamíferos. Por ejemplo, en el caso de humanos y otros primates, el saco vitelino secundario y la placenta temprana son estructuras anatómicas individualizadas (Gulbis et al., 1998). El sistema dinámico de la unidad feto-placenta produce un constante intercambio de líquidos y constituyentes del fluido, lo cual se refleja en cambios en los constituyentes bioquímicos, químicos y físicos de los fluidos fetales (Aidasani et al., 1993). Así como sucede en otras especies de mamíferos, en los camélidos sudamericanos, el desarrollo del feto, rodeado por el compartimento del fluido amniótico y conectado con el saco alantoideo vía el uraco y la vasculatura placental, recibe los nutrientes, principalmente por la vena umbilical (Battaglia y Meschia, 1988). Aidasani et al. (1992) reportaron que la composición de los fluidos fetales en mamíferos está influenciado por la excreción de orina fetal y que los cambios en la concentración de muchos componentes durante la preñez tardía pueden reflejar la actividad metabólica fetal (Prestes et al., 2001). 
El presente estudio es pionero en determinar los parámetros bioquímicos sanguíneos en las diferentes etapas de la gestación en camélidos sudamericanos (Cuadro 2), así como en evaluar la relación entre los parámetros bioquímicos de las madres y fetos en alpacas (Cuadro 1).

Los niveles de glucosa resultaron en una correlación positiva alta entre los valores séricos maternal y fetal evidenciándose un mayor nivel de glucosa sanguínea en sangre fetal que en la materna (Huaynates et al., 2016), similar a lo encontrado en bovinos y caprinos. En estas especies se evaluaron los niveles de glucosa en fluido amniótico (37.5 $\mathrm{mg} / \mathrm{dl}$ ) y alantoideo $(34.5 \mathrm{mg} / \mathrm{dl})$ mas no en suero fetal, habiendo encontrado $116 \mathrm{mg} / \mathrm{dl}$ de glucosa maternal (Tabatabaei, 2012; Tabatabaei y Mamoei, 2012). Es posible que los mayores requerimientos de glucosa fetal durante los últimos meses de gestación sean para garantizar el desarrollo del feto. De forma natural, las alpacas y llamas tienen mayores niveles de glucosa en sangre que otros mamíferos y en el presente estudio los niveles de glucosa sanguínea materno-fetal en alpacas se incrementaron progresivamente durante todo el periodo de gestación (Huaynates et al., 2016), similar a lo reportado en bovinos con cambios notorios a partir de la segunda mitad de la gestación (Tabatabaei y Mamoei, 2012).

En otras especies se ha encontrado variabilidad en los resultados de la glucosa sanguínea. En caprinos se reportan incrementos en la segunda mitad y etapa final de la gestación (Tabatabaei, 2012; Waziri et al., 2010) y en ovinos se ha descrito que el pico de glucosa plasmática ocurre en el último día de preñez (Charismiadou et al., 2000; Khatun et al., 2011) o sin cambios notorios (Balikci et al., 2007). Por otro lado, en marranas no se han observado diferencias significativas para los niveles de glucosa sanguínea durante la gestación (Žvorc et al., 2006).

Los niveles de triglicéridos (Cuadro 1) resultaron en una correlación positiva alta entre los valores séricos maternal y fetal, tal como sucede en ovejas durante la preñez tardía, donde los perfiles lípidos séricos están caracterizados por concentraciones incrementadas de colesterol total, triglicéridos y lipoproteínas (Schlumbohm et al., 1997). Los niveles de triglicéridos se incrementaron a lo largo de la gestación en alpacas, similar a lo reportado en borregas donde se observa un incremento a la mitad del periodo de preñez, aunque en otros estudios se muestra una disminución progresiva en los niveles de triglicéridos, sobre todo al final de la gestación (Balikci et al., 2007; Piccione et al., 2009). El incremento en los niveles de triglicéridos durante la gestación en alpacas podría deberse al efecto que ejerce la insulina, la cual juega un rol directo en el metabolismo del tejido adiposo, similar a lo encontrado en especies relacionadas como el ovino (Balikci et al., 2007).

Los niveles de colesterol (Cuadro 1) muestran una correlación positiva alta, tal y como se presenta en rumiantes menores durante la preñez tardía, donde los perfiles lipídicos séricos están caracterizados por concentraciones incrementadas de colesterol y otros lípidos. Por ejemplo, en borregas se encuentran niveles de colesterol de 57 a 83 $\mathrm{mg} / \mathrm{dl}$ y en el caso de triglicéridos 22 a 87 $\mathrm{mg} / \mathrm{dl}$ (Schlumbohm et al., 1997; Balikci et al., 2007; Piccione et al., 2009). A diferencia de lo reportado en el presente estudio, los bovinos presentan una disminución en los niveles de colesterol a lo largo de la gestación, reportándose valores decrecientes desde 126 a $85.5 \mathrm{mg} / \mathrm{dl}$ (Tabatabaei y Mamoei, 2012). Las cabras, asimismo, presentan una disminución significativa entre la primera etapa de la gestación, comparada con el resto de la gestación donde los valores se mantenían relativamente constantes por debajo de los 90 $\mathrm{mg} / \mathrm{dl}$ (Tabatabaei, 2012).

Los niveles de proteínas totales resultaron en una correlación positiva baja entre los valores séricos maternales y fetales (Cuadro 1), habiendo un aumento progresivo en suero fetal en comparación con los valores en sue- 
ro maternal de alpaca, donde se mantuvieron relativamente constantes a lo largo de la gestación (Cuadro 2). En el caso de bovinos y caprinos se reportan valores contradictorios, con incrementos significativos en la última fase de gestación (Tabatabaei y Mamoei, 2012) y valores constantes a lo largo de la gestación (Waziri et al., 2010), así como disminución de los niveles en el último tercio gestacional en el caso de borregas (Antunovic et al., 2002; Balikci et al., 2007; Piccione et al., 2009).

En los niveles de calcio materno-fetal se pudo observar un incremento significativo en el último tercio de gestación (Cuadro 2); sin embargo, la correlación materno-fetal fue nula (Cuadro 1). Estudios en ovejas muestran una tendencia al incremento de calcio en preñez avanzada, con una razón de absorción de calcio de al menos $115 \mathrm{mg} / \mathrm{kg}$ de peso corporal (Sikes y Dingwall, 1975; Kadzere et al., 1996). De acuerdo a Kadzere et al. (1996), los requerimientos de calcio en esta etapa y durante la lactación son mayores que los requerimientos en cualquier otra etapa de la vida de las hembras, incrementándose la absorción de calcio desde el tracto gastrointestinal. Una explicación de los altos niveles de calcio en esta etapa se atribuye al incremento plasmático de la hormona paratiroidea (Georgievskii et al., 1982), la cual activa los osteoclastos e incrementan los niveles de calcio de las reservas del animal.

Los niveles de bilirrubina en sangre materna durante toda la etapa de gestación se describen en muy pocos estudios, siendo uno de los más actuales el trabajo de Balikci et al. (2007) en borregas donde reportan un incremento de bilirrubina materna, sobre todo en la última fase de la gestación, a diferencia de lo hallado en el presente estudio, donde no se encontraron cambios significativos en los niveles de bilirrubina total materno-fetal, salvo cambios en los niveles de bilirrubina directa maternal en el último tercio de gestación. Esto último sería debido a la bilirrubina adicional derivada de la degradación de la hemoglobina fetal o por una inadecuada síntesis del ácido glucorónico.
La fosfatasa alcalina mostró correlación positiva baja para los niveles materno-fetales (Cuadro 1), pero sin diferencias significativas entre las etapas de gestación maternal o fetal (Cuadro 2). Estos resultados difieren a lo reportado en bovinos y caprinos donde se encontraron incrementos de fosfatasa alcalina a lo largo de la gestación (Tabatabaei, 2012; Tabatabaei y Mamoei, 2012). Es conocida la estrecha relación entre los valores de calcemia y fosfatasa alcalina en sangre, de allí que es probable que los niveles de fosfatasa alcalina se mantengan constantes a lo largo de la gestación en alpacas, debido principalmente a la falta de cambios significativos en la calcemia encontrada en el presente estudio.

\section{Conclusiones}

- Existe correlación positiva entre los niveles de glucosa, colesterol, triglicéridos, fosfatasa alcalina, proteínas totales y albúmina obtenidos en suero materno-fetal de alpacas.

- Los niveles de colesterol, triglicéridos, fosfatasa alcalina, proteínas totales y albúmina fueron superiores en suero materno que en suero fetal.

- Los niveles de glucosa sanguínea fetal fueron superiores a los encontrados en suero maternal.

- Los niveles de bilirrubina total, fosfatasa alcalina, proteínas totales y albúmina se mantuvieron constantes durante todo el periodo de gestación.

\section{Literatura Citada}

1. Aidasani R, Chauhan RAS, Tiwari S, Shukla SP. 1992. Some metabolic constituents of caprine foetal fluids and foetal serum. Indian J Anim Sci 62:335336.

2. Aidasani R, Chauhan RAS, Tiwari S, Shukla SP. 1993. Studies on electrolytes of caprine foetal fluids and foetal serum. Indian Vet J 70: 239-241. 
3. Antunovic Z, Sencic D, Speranda M, Liker B. 2002. Influence of the season and the reproductive status of ewes on blood parameters. Small Rumin Res 45: 39-44. doi: 10.1016/S0921-4488(02) 00109-8

4. Balikci E, Yildiz A, Gurdogan F. 2007. Blood metabolite concentrations during pregnancy and postpartum in Akkaraman ewes. Small Rum Res 67: 247-251. doi: 10.1016/j.smallrumres. 2005.10.011

5. Battaglia FC, Meschia G. 1988. Fetal nutrition. Ann Rev Nutr 8: 43-61. doi: 10.1146/annurev.nu.08.070188.000355

6. Brozostowski H, Milewski S, Wasilewska A, Tanski Z. 1995. The influence of the reproductive cycle on levels of some metabolism indices in ewes. Arch Vet Pol 35: 53-62.

7. Charismiadou MA, Bizelis JA, Rogdakis E. 2000. Metabolic changes during the perinatal period in dairy sheep in relation to level of nutrition and breed. I. Late pregnancy. J Anim Physiol Anim Nutr 84: 61-72. doi: 10.1046/j.14390396.2000.00282.x

8. El-Sherif MM, Assad F. 2001. Changes in some blood constituents of Barki ewes during pregnancy and lactation under semiarid conditions. Small Rum Res 40: 269-277. doi: 10.1016/S0921-4488(01) 00174-2

9. Fýrat A, Ozpýnar A. 2002. Metabolic profile of pre-pregnancy, pregnancy and early lactation in multiple lambing Sakýz ewes. 1. Changes in plasma glucose, 3hydroxybutyrate and cortisol levels. Ann Nutr Metab 46: 57-61.

10. Georgievskii VI, Annenkov BN, Samokhin VT. 1982. Mineral nutrition of animals. London: ButterworthHeinemann. 475 p.

11. Gulbis B, Jauniaux E, Cotton F, Stordeur P. 1998. Protein and enzyme patterns in the fluid cavities of the first trimester gestational sac: relevance to the absorptive role of secondary yolk sac. Mol Hum Reprod 4: 857-862. doi: 10.1093/molehr/4.9.857
12. Huaynates J, Espinoza J, López-Torres B, Rodríguez A, Caro $C$, Rodríguez J. 2016. Relación entre la glucemia maternal y fetal y el páncreas endocrino fetal en alpacas. Rev Inv Vet Perú 27: 17-23. doi: 10.15381/rivep. v27i1.11463

13. Iriadam M. 2007. Variation in certain haematological and biochemical parameters during the peri-partum period in Kilis does. Small Rum Res 73: 54-57. doi: 10.1016/j.smallrumres.2006.11.001

14. Kadzere CT, LLewelyn CA, Chivandi E. 1996. Plasma progesterone, calcium, magnesium and zinc concentrations from oestrus synchronization to weaning in indigenous goats in Zimbabwe. Small Rum Res 24: 21-26. doi: 10.1016/S09214488(96)00933-9

15. Karapehlivan M, Atakisi E, Atakisi O, Yucayurt R, Pancarci SM. 2007. Blood biochemical parameters during the lactation and dry period in Tuj ewes. Small Rum Res 73: 267-271. doi: 10.1016/j.smallrumres.2006.12.006

16. Khatun A, Wani GM, Bhat JIA, Choudhury AR, Khan MZ. 2011. Biochemical indices in sheep during different stages of pregnancy. Asian J Anim Vet Adv 6: 175-181. doi: 10.3923/ ajava.2011.175.181

17. Krajnicakova M, Kovac G, Kostecky M, Valocky I, Maracek I, Šutiakova I, Lenhardt L. 2003. Selected clinicobiochemical parameters in the puerperal period of goats. Bull Vet Inst Pulawy 47: 177-182.

18. Nazifi S, Saeb M, Ghavami SM. 2002. Serum lipid profile in Iranian fat-tailed sheep in late pregnancy, at parturition and during the post-parturition period. J Vet Med A Physiol Pathol Clin Med 49: 912. doi: $10.1046 / j .1439-0442.2002$. 00405.x

19. Piccione G, Caola G, Giannetto C, Grasso F, Runzo SC, Zumbo A, Pennisi P. 2009. Selected biochemical serum parameters in ewes during pregnancy, post-parturition, lactation and dry period. Anim Sci Pap Rep 27: 321-330. 
20. Prestes NC, Chalhoub MC, Lopes MD, Takahira RK. 2001. Amniocentesis and biochemical evaluation of amniotic fluid in ewes at 70, 100 and 145 days of pregnancy. Small Rumin Res 39: 277-281. doi: 10.1016/S0921-4488(00) 00202-9

21. Rodríguez J, Cueva S, Lira B, Espinoza J, Vásquez M. 2012. Identificación inmunohistoquímica de transportadores de glucosa intestinal y absorción de glucosa durante el desarrollo y maduración del intestino delgado de crías de alpacas. Rev Inv Vet Perú 23: 126137. doi: 10.15381/rivep.v23i2.892

22. Schlumbohm C, Sporleder HP, Gurtler H, Harmeyer J. 1997. The influence of insulin on metabolism of glucose, free fatty acids and glycerol in normo- and hypocalcemic ewes during different reproductive states. Deut Tierarz Wochen 104: 359-365.

23. Swanson KS, Kuzmuk KN, Schook LB, Fahey GC Jr. 2004. Diet affects nutrient digestibility, haematology, and serum chemistry of senior and weanling dogs. J Anim Sci 82: 1713-1724.

24. Sykes AR, Dingwall RA. 1975. Calcium absorption during lactation in sheep with demineralised skeletons. J Agric Res Sci
84: 245-248. doi: 10.1017/S0021859600052357

25. Tabatabaei S. 2012. Gestational variations in the biochemical composition of the fetal fluids and maternal blood serum in goat. Comp Clin Pathol 21: 1305-1312. doi: 10.1007/s00580-0111286-4

26. Tabatabaei S, Mamoei M. 2012. Changes in the biochemical composition of fetal fluids and maternal blood serum during different days of gestation in cattle. Comp Clin Pathol 21: 1005-1012. doi: 10.1007/s00580-011-1217-4

27. Yokus B, Cakir DU, Kanay Z, Gulten T, Uysal E. 2006. Effects of seasonal and physiological variations on the serum chemistry, vitamins and thyroid hormone concentrations in sheep. J Vet Med A Physiol Pathol Clin Med 53: 271-276. doi: 10.1111/j.1439-0442.2006.00831.x

28. Žvorc Z, Mrljak V, SuJiè V, Gotal JP. 2006. Hematological and biochemical parameters during pregnancy and lactation in sows. Vet Arhiv 76: 245-253.

29. Waziri MA, Ribadu AY, Sivachelvan $N$. 2010. Changes in the serum proteins, hematological and some serum biochemical profiles in the gestation period in the Sahel goats. Vet Arhiv 80: 215-224. 